\title{
Liposomal Hydrogel Formulation for Transdermal Delivery of Pirfenidone
}

Anup Jose, Praveen Kumar Mandapalli, Venkata Vamsi Krishna Venuganti*

\section{Supplementary material}

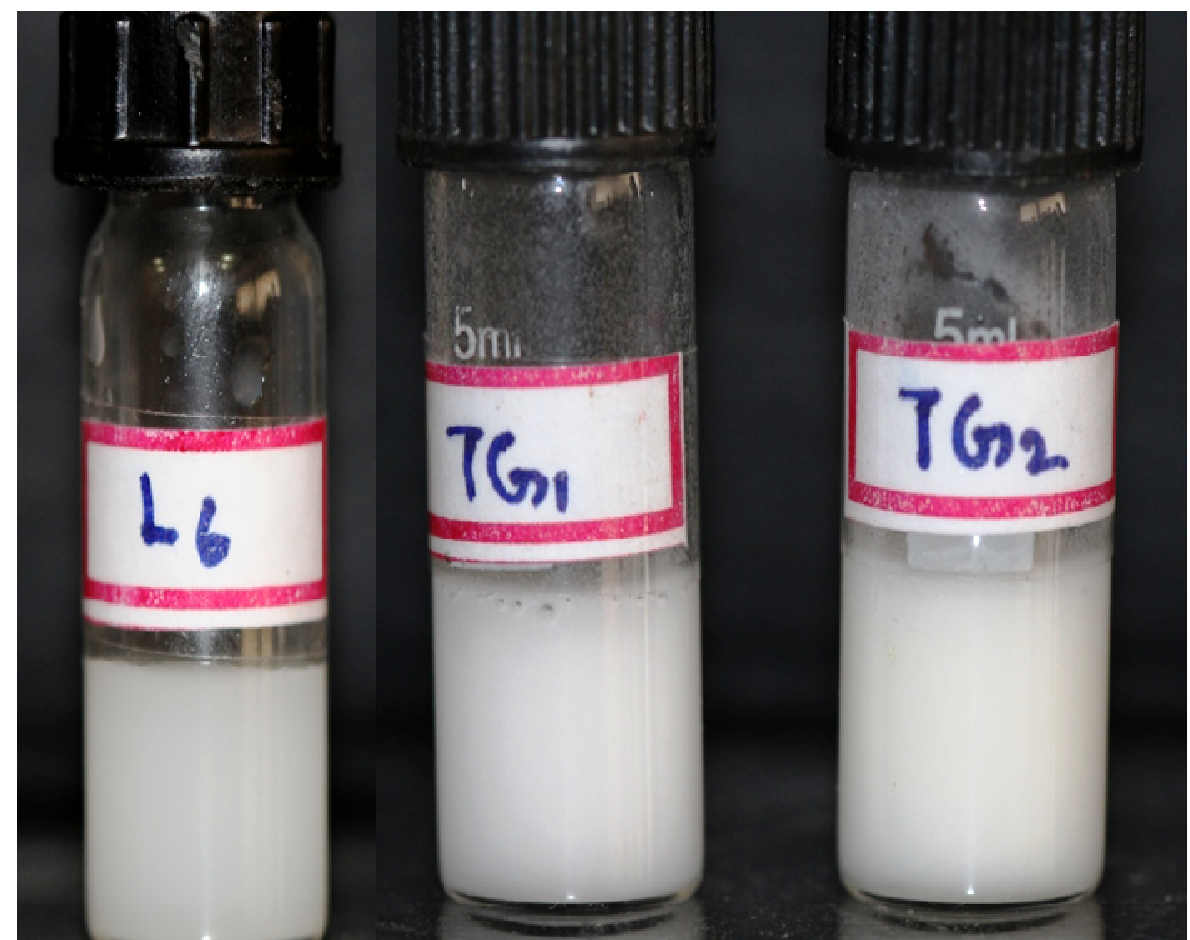

A

B

C

Figure S1: Photographs of PFD loaded liposomes (A, $10 \mathrm{mg} / \mathrm{mL}$ lipid composition), PFD loaded liposomes incorporated in hydrogel containing propylene glycol and oleic acid (B), PFD loaded liposomes incorporated in hydrogel containing IPM and oleic acid (C). 


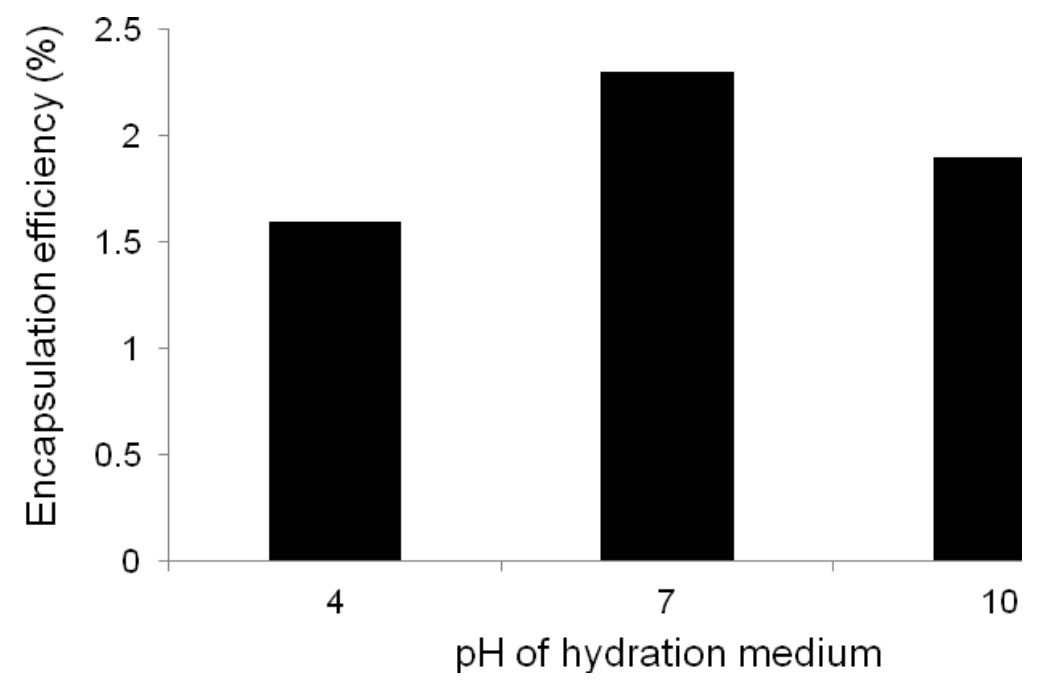

Figure S2. Effect of hydration medium pH on encapsulation efficiency of PFD in liposomes. 